\title{
Migração do fio de Kirschner após o tratamento da luxação acromioclavicular para o ombro contralateral - relato de caso*
}

\section{Kirschner Wire Migration after the Treatment of Acromioclavicular Luxation for the Contralateral Shoulder - Case Report}

\author{
Fabiano Rogerio Palauro ${ }^{1}$ Guilherme Augusto Stirma ${ }^{1}$ Armando Romani Secundino ${ }^{1}$ \\ Gabriel Bonato Riffel ${ }^{1}$ Filipe Baracho ${ }^{1}$ Leonardo Dau ${ }^{1}$ \\ ${ }^{1}$ Departamento de Ortopedia e Traumatologia, Universidade Federal \\ do Paraná (UFPR), Curitiba, PR, Brasil \\ Rev Bras Ortop 2019;54:202-205. \\ Address for correspondence Guilherme Augusto Stirma, \\ Departamento de Ortopedia e Traumatologia, Universidade Federal \\ do Paraná (UFPR), Curitiba, PR, Brasil \\ (e-mail: guilhermeaugusto89@hotmail.com).
}

\section{Resumo \\ Palavras-chave \\ - migração de corpo estranho \\ - luxação do ombro \\ - articulação do ombro \\ - articulação acromioclavicular \\ - fios ortopédicos}

\begin{abstract}
\footnotetext{
Trabalho desenvolvido no Departamento de Ortopedia e Traumatologia, Universidade Federal do Paraná (UFPR), Curitiba, PR, Brasil. Publicado originalmente por Elsevier Ltda.
} com sucesso.
O uso dos fios metálicos, denominados fios de Kirschner, é um método de fixação simples e eficaz para a correção de fraturas e luxações do ombro na cirurgia ortopédica. Uma das possíveis complicações é a migração do fio durante o acompanhamento pósoperatório. Os autores apresentam um caso de um paciente masculino de 48 anos, administrador, que sofreu uma queda de mesmo nível com trauma em ombro direito durante uma partida de futebol. Atendido em um hospital de referência de ortopedia e traumatologia, foi diagnosticada luxação acromioclavicular grau V. Quatro dias após o trauma, fez-se o tratamento cirúrgico da luxação acromioclavicular com amarrilhos com fios de âncora, transferência do ligamento coracoacromial e fixação com fio de Kirchner do acrômio à clavícula. No retorno, 12 dias após o procedimento cirúrgico, identificou-se a migração do fio de Kirschner do bordo do acrômio. Apesar de orientado a se submeter a cirurgia para remoção do fio, o paciente se recusou. Nove meses após o tratamento cirúrgico, o paciente apresentou dores no ombro esquerdo (lado contralateral), dificuldade para mobilizar o ombro, equimose e saliência. Foram feitas radiografias bilaterais e foi constatado que o fio de Kirschner, originalmente no ombro direito, estava no ombro contralateral. Fez-se então cirurgia para remoção do implante,

The use of metal wires, called Kirschner wires, is a simple and effective fixation method for the correction of shoulder fractures and of dislocations in orthopedic surgery. Wire migration during the postoperative follow-up is a possible complication of the procedure. The authors present the case of a 48-year-old male patient, a business received

July 31,2017

accepted

September 13, 2017

published online

April 15, 2019
DOI https://doi.org/

10.1016/j.rbo.2017.09.017. ISSN $0102-3616$.
Copyright $(2019$ by Sociedade Brasileira License terms de Ortopedia e Traumatologia. Published by Thieme Revnter Publicações Ltda, Rio de Janeiro, Brazil 


\author{
Keywords \\ - foreign-body \\ migration \\ - shoulder dislocation \\ - shoulder joint \\ - acromioclavicular \\ joint \\ - bone wires
}

administrator, who suffered a fall from his own height during a soccer match resulting in right shoulder trauma. The patient was treated at a specialized orthopedics and trauma hospital and was diagnosed with a grade $V$ acromioclavicular dislocation. Four days after the trauma, the acromioclavicular dislocation was surgically treated using ligatures with anchor wires, coracoacromial ligament transfer, and fixation with Kirshner wires from the acromion to the clavicle. At the follow-up, 12 days after the surgical procedure, migration of the Kirschner wire to the acromion edge was identified. The patient was oriented to undergo another surgery to remove the Kirshner wire, due to the possibility of further migration; nonetheless, he refused the surgery. Nine months after the surgical treatment, the patient complained of pain on the left shoulder (contralateral side), difficulty to mobilize the shoulder, ecchymosis, and protrusion. Bilateral radiographs demonstrated that the Kirschner wire, originally from the right shoulder, was on the left side. The patient then underwent a successful surgery to remove the implant.

\section{Introdução}

O uso de fios metálicos para a cirurgia ortopédica foi introduzido por Martin Kirschner no início do século XX, é um método de fixação simples e eficaz para a correção de fraturas e luxações do ombro na cirurgia ortopédica. Entre suas complicações incluíam-se dano vascular e nervoso, rupturas tendinosas, osteomielite, perda de redução da fratura ou luxação, infecção superficial e migração do fio durante o acompanhamento pós-operatório. ${ }^{1-3}$

A migração dos fios pode acarretar efeitos devastadores, ocasionar em um aumento da morbidade e mortalidade. Já foram encontrados e descritos os fios metálicos em pulmões, esôfago, artérias (aorta, braquiocefálica, subclávia), veias, mediastino, coração, medula espinhal em sua porção cervical, fígado e baço. $O$ mecanismo que causa a migração ainda é desconhecido, embora a atividade muscular seja postulada como uma das possíveis causas. ${ }^{2-5}$

Assim, o objetivo deste estudo é relatar o caso em que se tratou uma luxação acromioclavicular com fios metálicos e amarrilhos, no entanto durante o acompanhamento ocorreu a migração do fio de Kirschner para o ombro contralateral.

\section{Relato de caso}

Paciente masculino de 48 anos, administrador, sofreu durante uma partida de futebol uma queda de mesmo nível com trauma em ombro direito. Atendido em um hospital de referência de ortopedia e traumatologia, foi diagnosticado com uma luxação acromioclavicular grau $V$.

Após quatro dias (- Fig. 1) do trauma fez-se o tratamento cirúrgico da luxação acrômio-clavicular com amarrilhos com fios da âncora, transferência do ligamento coracoacromial e fixação com fio de Kirchner do acrômio à clavícula.

No retorno após 12 dias do procedimento cirúrgico, identificou-se a migração do fio de Kirschner ao rebordo do acrômio. Informou-se ao paciente da necessidade de acompanhamento com maior frequência e que em 45 dias a partir da cirurgia o fio seria retirado. Nos retornos de 20 e
30 dias, a radiografia permaneceu semelhante à de 12 dias sem avanços da migração.

Em 42 dias após a cirurgia o fio não estava saliente, o paciente não apresenta queixas álgicas, o arco de movimento do ombro estava preservado e amplo. 0 paciente foi enfaticamente orientado da necessidade de retirada do fio de Kirschner pela possibilidade de migração e pelo término do tratamento, no entanto negou-se veementemente, mas concordou em acompanhamento frequente ambulatorial.

Durante o acompanhamento de três, quatro e cinco meses a radiografia permaneceu sem evolução. Não apresentava queixas, força muscular grau $\mathrm{V}$, movimentos amplos.

Em seis meses não retornou conforme solicitado. Após nove meses do tratamento cirúrgico, iniciou com dores no ombro esquerdo (lado contralateral), dificuldade para mobilizar no arco de movimento, equimose e saliência (-Figs. 2 e 3 ). Radiografou-se bilateralmente e constatou-se que o fio de Kirschner originalmente do ombro direito estava no esquerdo (-Figs. 4 e 5 ).

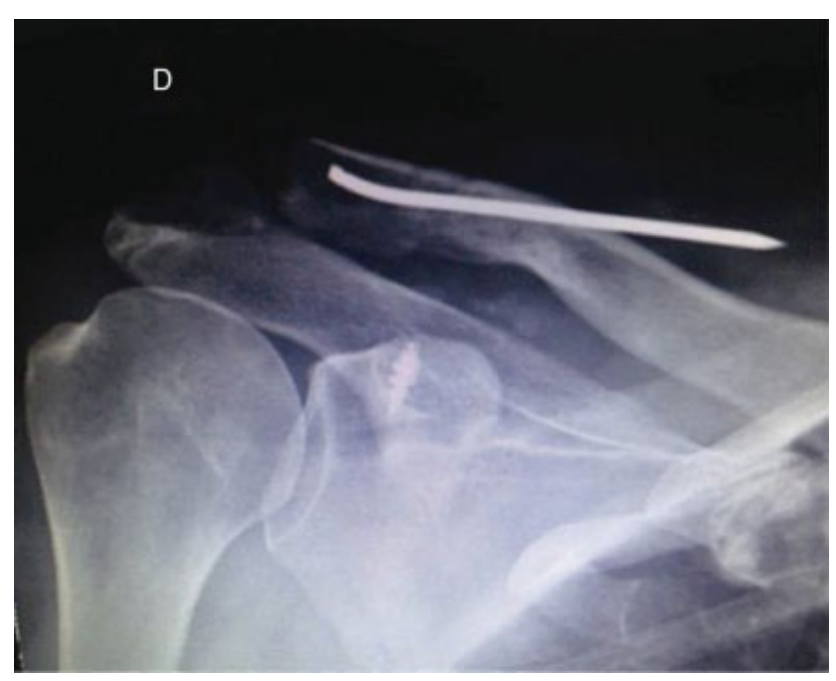

Fig. 1 Pós-operatório de 15 dias, ombro direito. 


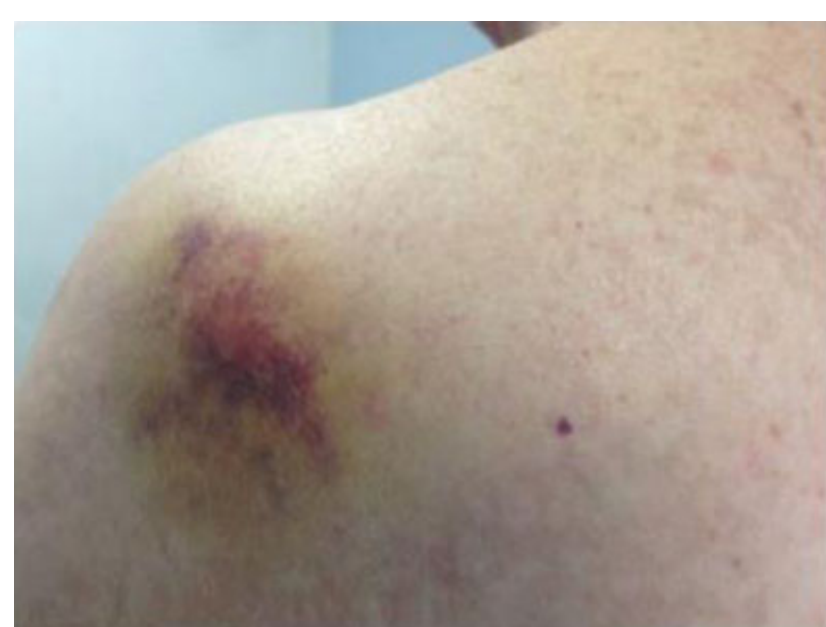

Fig. 2 Hematoma e uma pequena saliência na pele, ombro esquerdo, nove meses de pós-operatório.

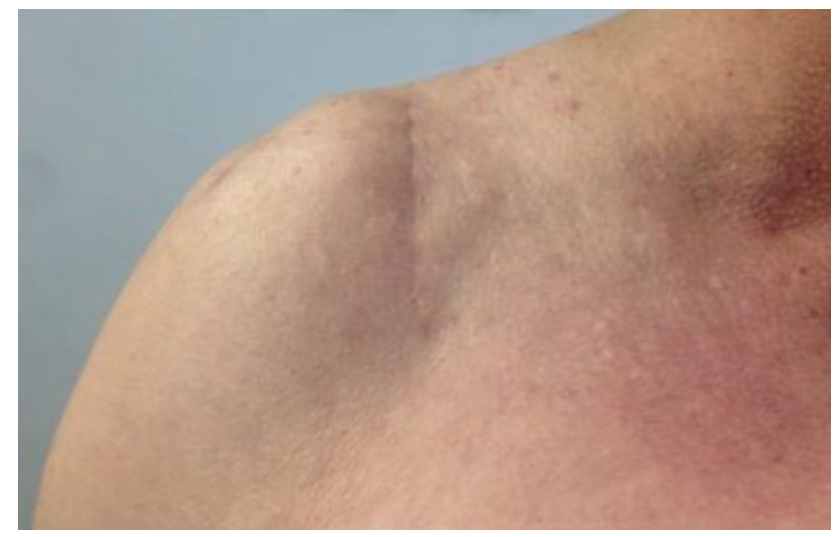

Fig. 3 Acesso cirúrgico, ombro direito, nove meses de pósoperatório.

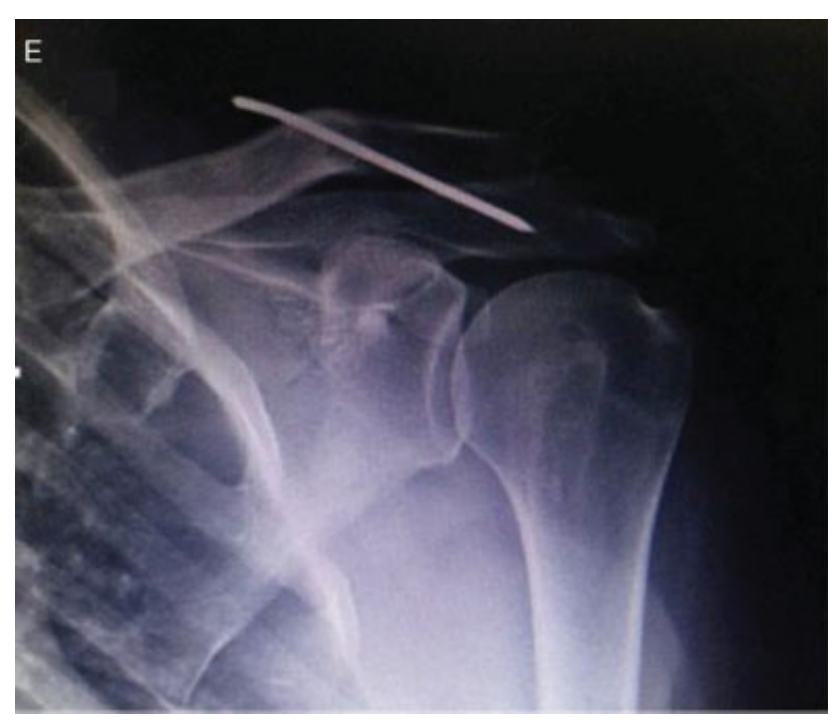

Fig. 4 Nove meses de pós-operatório, ombro esquerdo.

O paciente foi perguntado sobre sintomas adicionais e mencionou que nas semanas anteriores sentira desconforto na região posterior da cervical.

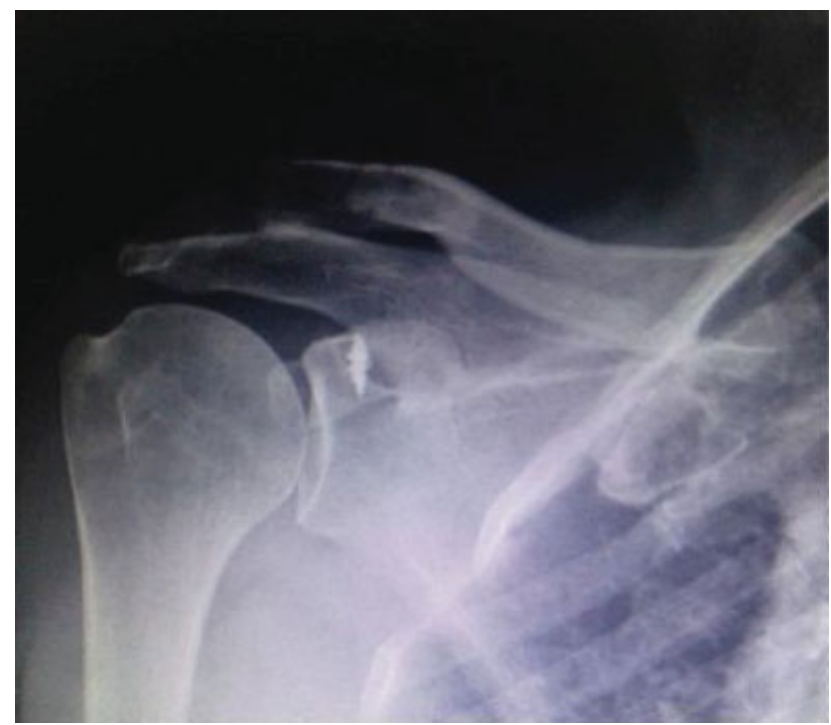

Fig. 5 Nove meses de pós-operatório, ombro direito.

Assim, fez-se um novo procedimento cirúrgico para a retirada do fio metálico no ombro esquerdo.

\section{Discussão}

A luxação acromioclavicular apresenta várias técnicas cirúrgicas para o seu tratamento. $O$ uso do fio de Kirschner é uma opção amplamente aceita pela sua facilidade e fácil manuseio. Apesar da baixa morbidade, algumas complicações podem ser observadas. ${ }^{3}$ A migração do fio de Kirschner é pouco frequente, o primeiro relato de caso foi feito por Mazet, ${ }^{6}$ em que o fio metálico migrou para o pulmão.

Segundo a revisão de Ballas e Bonnel, ${ }^{7} 88$ casos foram descritos na literatura. A patologia mais prevalente para essa complicação foi a fixação para a fratura de clavícula, seguidos da luxação esternoclavicular e acromioclavicular. Os locais mais frequentes para a migração foram a cavidades torácica e artérias de grande calibre. Não foi observada em sua revisão a migração para o ombro contralateral. ${ }^{7}$

O período de detecção é amplo, em nosso caso observouse a migração com nove meses de evolução.

Dos casos, 56\%, segundo Tan et al, ${ }^{1}$ são diagnosticados em média de três meses, enquanto que em alguns pacientes observou-se em questão de horas e em outros, de anos.

As causas para a migração ainda permanecem obscuras. Várias teorias são propostas, inclusive a atividade muscular, a força gravitacional, a reabsorção óssea, a pressão negativa torácica, a técnica cirúrgica aplicada inadequadamente, o movimento respiratório e a ampla mobilidade do ombro também são descritos como possíveis causas, porém nosso paciente trabalhava como administrador, assim não exercia trabalho braçal. ${ }^{8}$

A evolução da migração do fio metálico pode ser trágica de extrema gravidade, acarretar procedimentos secundários complexos e em alguns casos até a morte do paciente. Particularmente se o fio metálico migrar para o sistema cardiovascular. ${ }^{1}$ Em alguns estudos sugere-se que deve ser contraindicado usar fios metálicos em cirurgias ortopédicas no ombro, pelo risco de vida após a migração intra-aórtica. ${ }^{2,9}$ Com base na revisão de 
Lyons, algumas recomendações precisam ser feitas durante e após a fixação com fios de Kirschner. Entre elas o uso desse método com extrema precaução, orientação do paciente para manter o retorno e acompanhamento para análise da evolução do caso, a extremidade distal do fio, próxima à pele, deve ser dobrada para atingir uma angulação próxima a 90o, sempre radiografar intraoperatoriamente ou no pós-operatório imediato para analisar a posição do fio e acompanhar com radiografias e clinicamente o paciente até a sua retirada, retirar os fios de Kirschner ao acabar o período de tratamento e ao se perceber sua migração independentemente da falta de sintomas clínicos. ${ }^{2}$

Durante este caso relatado houve o acompanhamento com radiografias seriadas, o fio metálico foi dobrado para dificultar sua migração e a orientação do paciente foi feita quando se percebeu a migração do fio de metálico e ao término do período de tratamento. Contudo, a dobra na extremidade distal do fio metálico não alcançou a angulação necessária para evitar a migração, o que possibilitou que essa complicação ocorresse.

\section{Conclusão}

O uso dos fios de Kirschner é um instrumento que auxilia no tratamento da luxação acromioclavicular. No entanto existem um risco considerável de migração para diversos locais, mesmo com acompanhamento cuidadoso, e a necessidade de retirá-lo assim que diagnosticada a migração.
Conflitos de interesse

Os autores declaram não haver conflitos de interesse.

\section{Referências}

1 Tan L, Sun DH, Yu T, Wang L, Zhu D, Li YH. Death due to intra-aortic migration of Kirschner wire from the clavicle: a case report and review of the literature. Medicine (Baltimore) 2016;95(21):e3741

2 Lyons FA, Rockwood CA Jr. Migration of pins used in operations on the shoulder. J Bone Joint Surg Am 1990;72(08):1262-1267

3 Batın S, Ozan F, Gürbüz K, Uzun E, Kayalı C, Altay T. Migration of a broken Kirschner wire after surgical treatment of acromioclavicular joint dislocation. Case Rep Surg 2016;2016:6804670

4 Fransen P, Bourgeois S, Rommens J. Kirschner wire migration causing spinal cord injury one year after internal fixation of a clavicle fracture. Acta Orthop Belg 2007;73(03):390-392

5 McCaughan JS Jr, Miller PR. Migration of Steinmann pin from shoulder to lung. JAMA 1969;207(10):1917

6 Mazet R. Migration of a Kirschner wire from the shoulder region into the lung: report of two cases. J Bone Joint Surg. 1943;25(02): 477-483

7 Ballas R, Bonnel F. Endopelvic migration of a sternoclavicular Kwire. Case report and review of literature. Orthop Traumatol Surg Res 2012;98(01):118-121

8 Freund E, Nachman R, Gips H, Hiss J. Migration of a Kirschner wire used in the fixation of a subcapital humeral fracture, causing cardiac tamponade: case report and review of literature. Am J Forensic Med Pathol 2007;28(02):155-156

9 Venissac N, Alifano M, Dahan M, Mouroux J. Intrathoracic migration of Kirschner pins. Ann Thorac Surg 2000;69(06):1953-1955 